\title{
Comparison of Endoscopic Retrograde Cholangiopancreatography (ERCP) and Magnetic Resonance Cholangiopancreatography (MRCP) in Bile Duct Imaging
}

\author{
Mehmet Ali Eryılmaz ${ }^{1}$, Ömer Karahan ${ }^{1}$, İsmet Tolü ${ }^{2}$, Ahmet Okuş ${ }^{1}$, Serden Ay ${ }^{2 *}$, Barış Sevinç ${ }^{1}$, \\ Ahmet Hakan Halıci ${ }^{1}$ \\ ${ }^{1}$ Konya Training and Research Hospital General Surgery Clinic, Konya, Turkey \\ ${ }^{2}$ Konya Training and Research Hospital Radiology Clinic, Konya, Turkey \\ Email: *serdenay@yahoo.com
}

Received August 10, 2012; revised September 11, 2012; accepted September 22, 2012

\begin{abstract}
Purpose: The aim of this study was to compare magnetic resonance cholangiopancreatography (MRCP) with endoscopic retrograde cholangiopancreatography (ERCP) in diagnosing bile duct pathologies. Materials and Methods: We documented the data of 171 patients with both ERCP and MRCP between January 2009 and December 2010 at the Konya Education and Research Hospital. Results: Of the 171 patients, $100(58.5 \%)$ were female and $71(41.5 \%)$ were male. The median age was 63 (55 to 89). ERCP was used to diagnose bile duct stones in $102(59 \%)$ patients, bile duct tumour in $14(8 \%)$ patients, hydatic cysts opening up to the bile duct in $4(2 \%)$ patients and bile duct stenosis in $3(1.8 \%)$ patients. For the detection of bile duct stones, MRCP had a sensitivity of $92 \%$, a specificity of $74 \%$ and a diagnostic accuracy of $83 \%$. For bile duct tumours, MRCP had a sensitivity of $85 \%$, a specificity of $98 \%$ and a diagnostic accuracy rate of $92 \%$. Conclusion: In our centre, the results of MRCP and ERCP were similar for the last two years. However, MRCP was superior with respect to diagnosis as it was cheaper and non-invasive. Thus, ERCP should be preferred for therapeutic processes.
\end{abstract}

Keywords: ERCP; MRCP; Bile Duct Disease; Diagnosis; Treatment

\section{Introduction}

In the diagnosis of bile duct pathologies, clinical laboratory findings and imaging studies play important roles. Frequently used imaging techniques are ultrasonography (USG), magnetic resonance cholangiopancreatography (MRCP) and endoscopic retrograde cholangiopancreatography (ERCP). Abdominal USG is useful in detecting bile duct morphology; however, its sensitivity in detecting the ethiology of bile duct obstruction is low. In the diagnosis of bile duct stones, USG must be combined with other imaging methods [1]. The advantages of MRCP are it is not invasive or radionuclear and provides 3D images [2]. ERCP is another way of detecting those pathologies. Although it is an invasive method, some therapeutic investigations like sphincterotomy, stone extraction and stent placement can be performed while performing a diagnostic study [3]. According to some authors, $10 \%$ of MRCP and $5 \%$ of ERCP performed in

"Corresponding author. our country are optimal [4]. Therefore, we aimed to detect the differences and the advantages of the two techniques by comparing their diagnostic accuracy, cost, time and morbidity in our hospital.

\section{Materials and Methods}

\subsection{Patients}

At the Konya Training and Research Hospital between January, 2009 and December 2010, 655 patients underwent MRCP, and 283 patients, ERCP, for bile duct pathologies. 171 patients who had both MRCP and ERCP were included in the study. MRCP was used on patients who had clinical jaundice symptoms with or without pain in the upper right quadrant of the abdomen, and laboratory results showing higher values than $\mathrm{GGT}=40 \mathrm{IU} / \mathrm{L}$, $\mathrm{ALP}=120 \mathrm{IU} / \mathrm{L}$, direct bilirubin $=0.2 \mathrm{mg} / \mathrm{dl}$, and USG-detected choledoch duct wider than $7 \mathrm{~mm}$, who had pre-diagnoses of bile duct disorders. Those who received diagnoses such as bile duct stones or stenosis, bile leakage, parasitosis, pancreatic tumors, papilla water tumors, 
underwent ERCP in order to perform treatment or biopsy. Age, gender, and details of MRCP and ERCP reports of the patients included in the study were obtained retrospectively from the hospital automation system.

\subsection{MRCP Technique}

MRCP was performed before ERCP. In order to have an empty stomach and a maximally full gall bladder, the patients didn't have any food for 6 hours before the procedure. The MRCP device was 1.5 Tesla and had 4 phase channels (Avanto, Simens Medical Systems, Germany). The patients were not sedated for the MRCP procedure. Total procedure duration was approximately 4-5 minutes. MRCP images were evaluated by an experienced radiologist (Figures 1 and 2). No contrast material was used to obtain the MRCP images. The Social Security Institution paid TL 65 for the MRCP procedure in 2010.

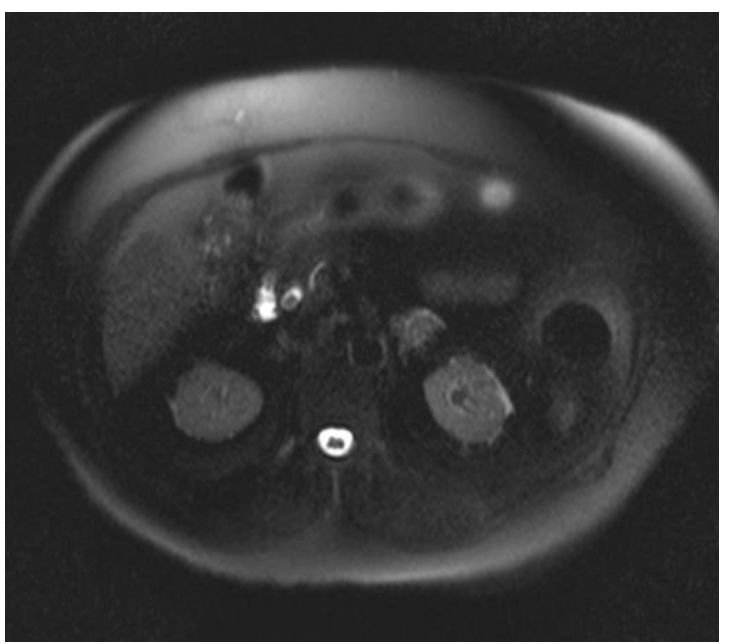

Figure 1. T2 axial MRCP image showing a bile duct stone.

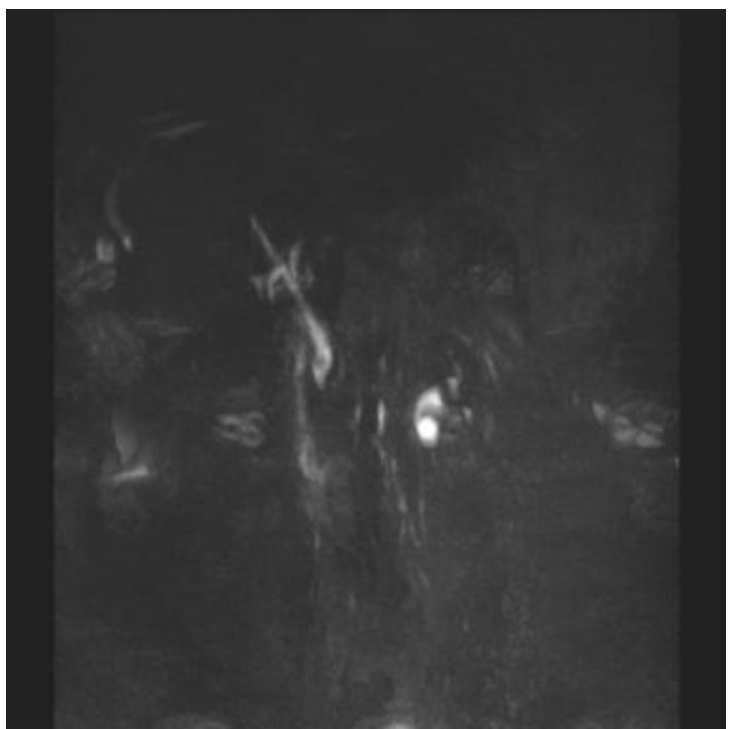

Figure 2. 3D MRCP image showing a bile duct stone.

\subsection{ERCP Technique}

ERCP was performed in order to confirm bile duct pathologies and to provide treatment, after informed consent was received from the patients. The equipment used during the ERCP procedures were C-Arm (Siemens, India), bipolar cautery (Olympus, Germany) and duodeno video endoscopy (Olympus, Japan). Every patient was admitted for treatment after fasting for eight hours. Parenteral Buscopan was administered to all patients. Endoscopic sphincterotomy (ES) and stone extraction were performed in the cases of stone, debris or parasitosis. In cases of tumoral lesions biopsy was performed for diagnostic confirmation, and stent placement was carried out where needed. The ERCP procedure took approximately 30 minutes. The Social Security Institution paid TL 284 for ERCP in 2010.

\subsection{Statistics}

Sensitivity, specifity, positive and negative predictive values and total accuracy rates were used. $P$ value was calculated by using SPSS (SPSS Inc., v16.0, 2009, Chicago, IL, USA). A P value of $<0.05$ was accepted as significant.

\section{Results}

One hundred $(58.5 \%)$ of the hundred and seventy one patients were female and $71(41.5 \%)$ were male. The median age was 63 years (55 to 89 ). In nine patients $(5.26 \%)$ the ERCP procedure could not be carried out. In six of these patients, the choledoch could not be cannulated, and 3 had pyloric stenosis. There was no failure during the MRCP procedures. The results of MRCP and ERCP in diagnosing bile duct disorders are given in Tables 1 and 2 .

Of the patients who had both MRCP and ERCP, MRCP detected bile duct stones in 103, and ERCP detected 102. Endoscopic sphincterotomy and stone extraction with ERCP were performed on these patients. Of the $32(19 \%)$ patients diagnosed by MRCP, ERCP found 26 $(15.20 \%)$ to have dilated bile ducts. However, neither method was able to distinguish pathology to explain the dilatation. ERCP detected tumors in 14 out of the 15 $(8.77 \%)$ patients diagnosed by MRCP with tumors, and biopsies were taken by ES. ERCP could not be performed on one patient due to inability to access the duodenum because of pyloric stenosis.

The diagnosis of 4 of the $5(2.92 \%)$ patients diagnosed by MRCP was confirmed by ERCP. ES was performed, and cystic vesicles and membranes removed. Four patients $(2.33 \%)$ were diagnosed with bile duct stenosis, two were treated with ES, and one with ES + balloon dilatation. Stent placement was performed on one. 
Table 1. MRCP and ERCP results of patients with bile duct disease.

\begin{tabular}{cccc}
\hline & MRCP n (\%) & ERCP n (\%) & P \\
\hline Unsuccessful investigation & 0 & $9(5)$ & $5(3)$ \\
Normal bile ducts & $6(4)$ & $102(59)$ & 0.002 \\
Bile duct stone & $103(60)$ & $26(15)$ & 0.912 \\
Bile duct dilatation (distal stenosis) & $32(19)$ & $14(8)$ & 0.387 \\
Bile duct tumor & $15(9)$ & $4(2)$ & 0.736 \\
Bile duct cystic disease & $5(3)$ & $3(1.7)$ & 0.703 \\
Bile duct stenosis & $4(2)$ & $4(2)$ & 0.410 \\
Bile duct inflammatory disease & $2(1.5)$ & $4(2)$ & 1 \\
Others & $4(2)$ & $171(100)$ & $1(100)$ \\
\hline
\end{tabular}

Table 2. Sensitivity test for MRCP.

\begin{tabular}{ccccc}
\hline & $\begin{array}{c}\text { Positive predictive } \\
\text { value }\end{array}$ & $\begin{array}{c}\text { Negative predictive } \\
\text { value }\end{array}$ & $\begin{array}{c}\text { Sensitivity } \\
(\%)\end{array}$ & \multicolumn{2}{c}{$\begin{array}{c}\text { Specifity } \\
(\%)\end{array}$} & 74 \\
Bile duct stone & 0.79 & 0.89 & 92 & 93 \\
Bile duct tumor & 0.80 & 0.98 & 85 & 98 \\
Bile duct cystic disease & 0.80 & 1 & 100 & 99 \\
Bile duct stenosis & 0.75 & 1 & 100 & 99 \\
\hline
\end{tabular}

Complications developed in $5(2.92 \%)$ of the cases that underwent stone extraction with ERCP. Two patients suffered duodenum perforation. Sphincteroplasty and primary repairs of the perforation were carried out through open surgery in one case. The other case recovered through conservative treatment. One patient developed hemorrhage, another developed pancreatitis. Both recovered through conservative treatment. The stone basket compacted in one patient. Both the basket wire and the stone were extracted with open surgery. Neither technique gave rise to mortality.

It was observed that ERCP and MRCP led to similarly accurate diagnoses, and there was no significant difference between the two techniques $(\mathrm{P}>0.05)$ (Tables 1 and 2).

\section{Discussion}

MRCP is an important diagnostic tool for biliary pathologies [4]. ERCP is an invasive procedure, and is used for treatment rather than diagnosis [3,5]. The diagnosis determined by MRCP can be confirmed by ERCP and/or surgical methods. In our study, 171 of the 655 patients that underwent MRCP underwent ERCP with the possible necessity of endoscopic treatment in mind. MRCP defined stones in the bile ducts of 103 patients. ERCP confirmed the presence of stones in 102 of those patients.
ES and stone extraction were performed. In one patient, the choledoch could not be cannulated due to diverticula in the duodenum. ES was performed on 14 patients that ERCP showed to have bile duct stenosis due to tumors, and biopsies were taken. In one case where a tumor in the papilla was observed along with stones, ES + stone extraction and biopsy were performed with ERCP. In one case, stent placement was performed to ensure bile flow (Table 1).

There are various studies investigating the sensitivities, specifities and precision of MRCP and ERCP in determining the pathologies of the bile ducts. In a prospective study by M. G. Scaffidi, et al., MRCP and ERCP results in determining choledoch stones were compared, and MRCP's sensitivity was found as $88 \%$, specifity as $72 \%$, and diagnostic accuracy as $83 \%$ [6]. In the study carried out by Lopez Hanninen, et al., on 66 patients, the sensitivity of MRCP in showing tumoral lesions of the bile ducts was reported as $83 \%$, its specifity as $96 \%$ and diagnostic accuracy as $90 \%$ [7]. In our study, in concordance with literature, the sensitivity of MRCP in identifying bile duct stones was determined as $92 \%$, its specifity as $74 \%$, diagnostic accuracy as $83 \%$, and in identifying tumoral lesions, its sensitivity as $85 \%$, specifity as 98\% and diagnostic accuracy as 92\% (Table 2).

Stenosis may develop in bile ducts due to prior surgery or 
to primary sclerosing cholangitis. According to literature, MRCP's sensitivity in showing the localization and length of the stenosis is $100 \%$ its specifity is $91 \%$, and diagnostic accuracy is $95 \%$ [8]. Our study results are similar. In this field, we found MRCP's sensitivity to be $100 \%$, specifity, 99\% and diagnostic accuracy, 99\%.

Bile duct obstruction due to hydatic cysts opening into the bile duct can be seen in $5 \%-25 \%$ of patients. ERCP and ES are one of the treatment methods [9]. In our study, hydatic cyst lesions opening into the bile duct were identified in $4(2.33 \%)$ patients. ES was performed by ERCP, and the vesicles and membranes of the cyst were removed. In our study, the sensitivity of MRCP was determined as $100 \%$, specifity as $99 \%$, and diagnostic accuracy as $99 \%$. There was no significant difference between MRCP and ERCP $(\mathrm{P}>0.05)$.

Stones that are smaller than the section thickness of the MR device may give false negative results, and in patients where there is a gap of more than 72 hours between the MRCP and the ERCP, although MRCP has indicated stones, ERCP may not find stones due to their spontaneous passing [10]. In such cases, ES is a therapeutical procedure [11]. In our study, 32 patients by MRCP and 26 patients by ERCP were detected to have dilated bile ducts that could not be explained by any obstructive pathology such as stones or tumors, and ES was performed.

MRCP and diagnostic ERCP were compared for cost effectiveness through a literature scan over 28 articles by Kaltenthaler et al., and it was decided that MRCP was more economical [12]. In our study, it was determined that the cost of one MRCP event cost TL 65, while one ERCP event cost approximately TL 284.

Even in experienced hands, ERCP has a cannulation failure rate of up to $3 \%-9 \%$ [13]. In our study, it has been observed that ERCP didn't give satisfactory results in $5.26 \%$ of the cases. ERCP, considered a reference method presently, is accepted to have a morbidity rate of $0.8 \%-10 \%$ [14]. Morbidity was $2.92 \%$ in our study. In one case where a bile duct stone was detected, the morbidity was due to basket compaction. Two cases had perforations, one had a hemorrhage and one had pacreatitis. T. Obana et al., have observed no mortalities connected to the ERCP procedure [15]. However, low that it may be, ERCP has a mortality rate of $0.7 \%-0.9 \%$ [16]. There were no mortalities in our study.

Our study has shown the ERCP procedures which are routinely carried out by gastroenterologists can be carried out successfully by experienced General Surgeons. 10\% of the MRCPs and 5\% of the ERCPs carried out in our country in the beginning of the 2000s were optimally successful [11]. In the 2010 plus years, MRCP can be carried out on all cases where clinicians deem it necessary without the need for sedation, and ERCP is carried out with success rates of up to $95 \%$ (Table 1).

Our study has some shortcomings. While MRCP was carried out on all patients with bile duct pathologies, selected patients underwent ERCP. ERCP and MRCP were not carried out on the same day. The diagnosis reached with MRCP was confirmed with ERCP. Surgery was performed in cases where ERCP was unsuccessful. In our country, the cost of MRCP and ERCP are based upon the unit price paid by the social security institution, and may differ from other countries.

\section{Conclusion}

In the identification of bile duct pathologies, MRCP has accuracy values near to that of ERCP. It is approximately four times more economical, and takes far less time. Therefore, MRCP should be used for diagnosis, and ERCP should be performed for treatment, if necessary.

\section{REFERENCES}

[1] E. Elüdokuz, E. Tankurt, H. Akbaylar, E. İğci, O. Dicle, İ. Şimşek, et al., "The Sensitivity of Ultrasonography in Cases Choledocholithiasis Diagnosed with ERCP," Turkiye Klinikleri Journal of Gastroenterohepatology, Vol. 7, 1996, pp. 108-110.

[2] P. D. Prabhakar, A. M. Prabhakar, H. B. Prabahakar and D. Sahani, "Magnetic Resonance Cholangiopancreatography of Benign Disorders of the Biliary System," Magnetic Resonance Imaging Clinics of North America, Vol. 18 , No. 3, 2010, pp. 497-514. doi:10.1016/i.mric.2010.08.007

[3] G. Ersöz, "Endoscopic Retrograde Cholangiopancreatography in Bile Duct Disorders," Türkiye Klinikleri Journal of Medical Sciences, Vol. 3, 2007, pp. 12-16.

[4] H. Şentürk, B. Canbakan and E. Özden, "ERCP: Indications and Risks," Series of Clinical Approach in Gastroenterology, Vol. 30, 2004, pp. 101-109.

[5] F. Maccioni, M. Martinelli, N. Al Ansari, A. Kagarmanova, et al., "Magnetic Resonance Cholangiography: Past, Present and Future: A Review," European Review for Medical and Pharmacological Sciences, Vol. 14, No. 8, 2010, pp. 721-725.

[6] E. C. Kaltenthaler, S. J. Walters, J. Chilcott, A. Blakeborough, Y. B. Vergel and S. Thomas, "MRCP Compared to Diagnostic ERCP for Diagnosis When Biliary Obstruction Is Suspected: A Systematic Review," BMC Medical Imaging, Vol. 6, 2006, pp. 9-24. doi:10.1186/1471-2342-6-9

[7] M. G. Scaffidi, C. Luigiano, P. Consolo, R. Pellicano, G. Giacobbe, M. Gaeta, et al., Magnetic Resonance Cholangiopancreatography versus Endoscopic Retrograde Cholangiopancreatography in the Diagnosis of Common Bile Duct Stones: A Prospective Comparative Study," Minerva Medica, Vol. 100, No. 5, 2009, pp. 341-348.

[8] E. L. Hänninen, H. Amthauer, N. Hosten, J. Ricke, M. Böhmig, J. Langrehr, et al., "Prospective Evaluation of Pancreatic Tumours: Accuracy of MR Imaging with MR 
Cholangiopancreatography and MR Angiography," Radiology, Vol. 224, No. 1, 2002, pp. 34-41. doi:10.1148/radiol.2241010798

[9] P. Angulo, D. H. Pearce, C. D. Johnson, J. J. Henry, N. F. LaRusso, B. T. Petersen, et al., "Magnetic Resonance Cholangiopancreatography in Patients with Biliary Disease: Its Role in Primary Sclerosing Cholangitis," Journal of Hepatology, Vol. 33, No. 4, 2000, pp. 520-527. doi:10.1016/S0168-8278(00)80002-1

[10] G. Galati, A. V. Sterpetti, M. Caputo, M. Adduci, G. Lucandri, S. Brozzetti, et al., "Endoscopic Retrograde Cholangiography for Intrabiliary Rupture of Hydatic Cyst," The American Journal of Surgery, Vol. 191, No. 2, 2006, pp. 206-210. doi:10.1016/j.amjsurg.2005.09.014

[11] M. M. Calvo, L. Bujanda, A. Calderon, J. L. Cabriada, A. Bernal, V. Orive, et al., "Role of Magnetic Resonance Cholangiopancreatography in Patients with Suspected Choledocholithiasis," Mayo Clinic Proceedings, Vol. 77, No. 5, 2002, pp. 422-428. doi:10.1016/S0025-6196(11)62210-6

[12] E. Kaltenthaler, Y. B. Vergel, J. Chilcott, S. Thomas, T. Blakeborough, S. J. Waters, et al., "A Systematic Review and Economic Evaluation of Magnetic Resonance Cholan- giopancreatography Compared with Diagnostic Endoscopic Retrograde Cholangiopancreatography," Health Technology Assessment, Vol. 8, No. 10, 2004, pp. 1-89.

[13] R. Schöfl, "Diagnostic Endoscopic Retrograde Cholangiopancreatography," Endoscopy, Vol. 33, No. 2, 2001, pp. 147-157. doi:10.1055/s-2001-11667

[14] M. L. Freeman, D. B. Nelson, S. Sherman, G. B. Haber, M. E. Herman, P. J. Dorsher, et al., "Complications of Endoscopic Biliary Sphincterotomy," The New England Journal of Medicine, Vol. 335, No. 13, 1996, pp. 909-919. doi:10.1056/NEJM199609263351301

[15] T. Obana, N. Fujita, Y. Noda, G. Kobayashi, K. Ito, J. Horaguchi, et al., "Efficacy and Safety of Therapeutic ERCP for the Elderly with Choledocholithiasis: Comparison with Younger Patients," Internal Medicine, Vol. 49, No. 18, 2010, pp. 1935-1941. doi:10.2169/internalmedicine.49.3660

[16] K. Hekimoğlu, Y. Üstündağ, A. Duşak, Z. Erdem, B. Karademir, S. Aydemir, et al., "MRCP vs ERCP in the Evaluation of Biliary Pathologies: Review of Current Literature," Journal of Digestive Diseases, Vol. 9, No. 3, 2008, pp. 162-169.

doi:10.1111/j.1751-2980.2008.00339.x 